Journal of Computer Science 3 (7): 556-560, 2007

ISSN 1549-3636

(C) 2007 Science Publications

\title{
A Block Cipher Using Linear Congruences
}

\author{
${ }^{1}$ V.U.K. Sastry and ${ }^{2}$ V. Janaki \\ ${ }^{1}$ Academic Affairs, Sreenidhi Institute of Science \& Technology, Ghatkesar, Hyderabad \\ ${ }^{2}$ Kakatiya Institute of Technology \& Science, Warangal, India
}

\begin{abstract}
We have developed a block cipher by using modular arithmetic inverse and linear congruences. The cipher contains a key matrix called the outer key. It also includes another key, which contains a set of constants involved in the linear congruences. This key is called as inner key. The cryptanalysis carried out in this paper indicates that the cipher cannot be broken by any cryptanalytic attack. This cipher is extended to the case of a larger block wherein interlacing and iteration also play a vital role.
\end{abstract}

Keywords: modular arithmetic inverse, inner key, outer key, linear congruences.

\section{INTRODUCTION}

In the literature of cryptography Hill cipher ${ }^{[1]}$ has been a prominent block cipher. Considering only 26 alphabetic characters a to $\mathrm{z}$, Hill developed a block cipher whose encryption can be described by the equation

$$
\mathrm{C}=\mathrm{KP} \bmod 26 \text {, }
$$

where $\mathrm{K}$ is a key matrix of size $n \times n, \mathrm{P}$ is a plaintext vector, and $\mathrm{C}$ is the ciphertext vector both having $\mathrm{n}$ components. The decryption of the cipher is carried out by using the relation

$$
\mathrm{P}=\mathrm{K}^{-1} \mathrm{C} \bmod 26
$$

where $\mathrm{K}^{-1}$ is the modular arithmetic inverse ${ }^{[2]}$ of the key matrix $\mathrm{K}$.

From the cryptanalysis of the cipher it is seen that it cannot be broken by bruteforce attack when the size of the matrix is large. However, in the case of the known plaintext attack, it is clearly established that the cipher can be broken by taking appropriately n column vectors of plaintext and ciphertext.

In the present paper our objective is to modify the Hill cipher by introducing an additional key. To this end, we use linear congruences, in the column vector of the plaintext, wherein the congruences contain the numbers corresponding to the plaintext characters. Here, we consider a plaintext, which includes characters that can be represented by ASCII code. Thus, we use mod 128 instead of mod 26 used in the Hill cipher. From the cryptanalysis developed in this paper, we find that the cipher cannot be broken by any cryptanalytic attack.

In section 2 of this paper, we have discussed the development of the cipher. In section 3, we have described the algorithms for encryption, decryption and presented a procedure for the modular arithmetic inverse of a matrix. In section 4 , we have illustrated the cipher by considering an example. The cryptanalysis for this cipher has been carried out in section 5.In section 6 , we have extended the analysis to a larger block by interlacing and iteration. Then, in section 7 , we have examined the avalanche effect. Finally in section 8 , we have presented the computations and conclusions.

\section{DEVELOPMENT OF THE CIPHER}

Consider a plaintext vector, which can be represented in the form

$$
\mathrm{p}=\left(\mathrm{p}_{1}, \mathrm{p}_{2}, \mathrm{p}_{3, \ldots \ldots \ldots \ldots \ldots \ldots \ldots \ldots \ldots} \mathrm{p}_{\mathrm{n}}\right)^{\mathrm{T}}
$$

Let us suppose that we choose a key matrix $\mathrm{K}$ given by

$$
\mathrm{K}=\left[\mathrm{K}_{\mathrm{ij}}\right], \mathrm{i}=1 \text { to } \mathrm{n}, \mathrm{j}=1 \text { to } \mathrm{n} \text {, }
$$

where the matrix $\mathrm{K}$ is non singular, and its determinant is relatively prime to 128 .

Corresponding Author: V. Janaki, Kakatiya Institute of Technology and Science, Warangal, India 
The aforementioned conditions are to be satisfied for the existence of the modular arithmetic inverse of $\mathrm{K}$ with respect to mod 128 .

Let $\mathrm{C}=\left(\mathrm{C}_{1}, \mathrm{C}_{2}, \mathrm{C}_{3} \ldots \ldots \ldots \ldots \ldots \ldots \mathrm{C}_{\mathrm{n}}\right)^{\mathrm{T}}$ be the ciphertext vector.

Let us now introduce $\mathrm{n}$ linear congruences given by

$$
\mathrm{P}_{\mathrm{i}}=\left(\mathrm{a}_{\mathrm{i}} \mathrm{p}_{\mathrm{i}}+\mathrm{b}_{\mathrm{i}}\right) \bmod 128, \mathrm{i}=1 \text { to } \mathrm{n},
$$

where $a_{i}$ and $b_{i}$ are constants, chosen appropriately, as mentioned below.

In the process of encryption, the ciphertext $\mathrm{C}$ can be written as

$$
\mathrm{C}=\mathrm{KP} \bmod 128
$$

where $\mathrm{P}=\left(\mathrm{P}_{1}, \mathrm{P}_{2}, \mathrm{P}_{3} \ldots \ldots \ldots \ldots \ldots \ldots \ldots \mathrm{P}_{\mathrm{n}}\right)^{\mathrm{T}}$.

Here the components of the plaintext vector $p_{i}$ ( $\mathrm{i}=1$ to $\mathrm{n}$ ) are obtained from the consecutive $\mathrm{n}$ characters of the given plaintext.

Here in (2.4), we choose each one of the $\mathrm{a}_{\mathrm{i}} \mathrm{s}$ as an odd integer, which lies between 0 and 127, and each one of the $b_{i} s$ as any integer lying between 0 and 127.The reason for this choice of the values of $a_{i}$ and $b_{i}$, will be clear very soon. When $\mathrm{a}_{\mathrm{i}}, \mathrm{b}_{\mathrm{i}}$ and $\mathrm{p}_{\mathrm{i}}$ are known to us we can readily calculate $P_{i}$ by using (2.4). On the other hand, when the $P_{i}$ is known to us, $p_{i}$ can be determined by solving (2.4). As we have assumed that $b_{i}$ is an integer which lies between 0 and 127, we can write (2.4) in the form

$$
\mathrm{P}_{\mathrm{i}}-\mathrm{b}_{\mathrm{i}}=\mathrm{a}_{\mathrm{i}} \mathrm{p}_{\mathrm{i}} \bmod 128 .
$$

As $a_{i}$ is an odd integer, which lies between 0 and 127, it is relatively prime to 128 .

Thus we obtain $d_{i}$, the multiplicative inverse if $a_{i}$, such that

$$
a_{i} d_{i} \bmod 128=1
$$

where $d_{i}$ is the multiplicative inverse of $a_{i}$.

From (2.6) and (2.7), we get

$$
\mathrm{p}_{\mathrm{i}}=\left(\mathrm{P}_{\mathrm{i}}-\mathrm{b}_{\mathrm{i}}\right) \mathrm{d}_{\mathrm{i}} \bmod 128 .
$$

Now let us consider the process of decryption. From the equation (2.5) we get

$$
\mathrm{P}=\mathrm{K}^{-1} \mathrm{C} \bmod 128
$$

where $\mathrm{K}^{-1}$ is the modular arithmetic inverse of $\mathrm{K}$. On using (2.9) and (2.8) we get $\mathrm{p}_{\mathrm{i}}$, the components of the plaintext as we have indicated in the aforementioned discussion.

The problem of the encryption, given by the equation (2.5) can be written in the form

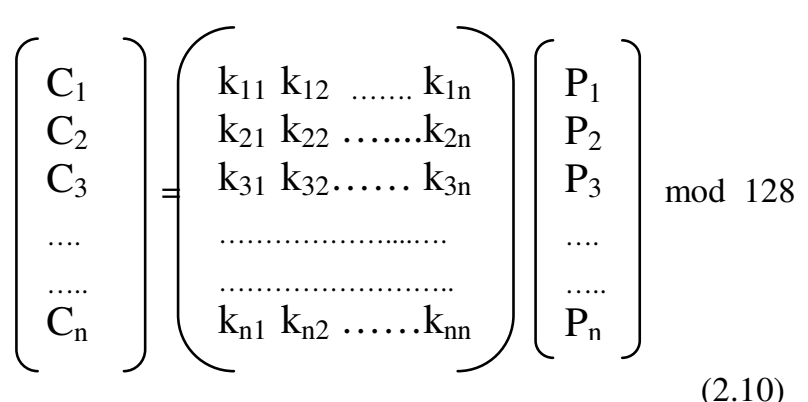

Here firstly we have to obtain $P_{i}$ where $P_{i}=\left(a_{i} p_{i}+b_{i}\right)$ mod 128.For this we require the values of $a_{i}$, and $b_{i}(i=1$ to $\mathrm{n})$.To this end we introduce a key comprising the numbers $a_{1}, a_{2} \ldots \ldots a_{n}$ and $b_{1}, b_{2} \ldots \ldots b_{n}$ and call this as the inner key. Subsequently we have to apply the key matrix $\mathrm{K}$ for obtaining $\mathrm{C}$. Thus we consider this key as the outer key. Here it is to be noted that in the process of decryption firstly the outer key is to be applied and then the inner key is to be used. Thus both the keys are to be supplied by the sender in a secret manner to the receiver.

In what follows we design the algorithms for the encryption, the decryption, and the modular arithmetic inverse of the key matrix.

\section{ALGORITHMS}

\subsection{Algorithm for encryption}

\{

1. for $\mathrm{i}=1$ to $\mathrm{n}$

\{

2. read $p_{i}, a_{i}, b_{i}$.

3. $P_{i}=\left(p_{i} a_{i}+b_{i}\right) \bmod 128$. \}

4. read the key matrix K.

5. $\mathrm{C}=\mathrm{KP} \bmod 128$.

6. write $\mathrm{C}$. \} 


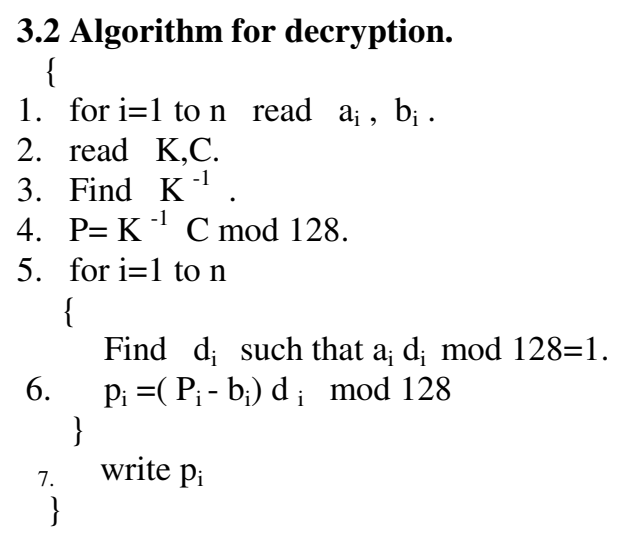

\subsection{Algorithm for modular arithmetic inverse}

// $\mathrm{A}$ is an nxn matrix. $\mathrm{N}$ is a positive integer with which //modular arithmetic inverse is carried out. Here $\mathrm{N}=128$. \{

1. Find the determinant of $\mathrm{A}$. Let it be denoted by $\Delta$, where $\Delta \neq 0$.

2. Find the inverse of $A$. The inverse is given by $\left[\mathrm{A}_{\mathrm{ji}}\right] / \Delta$.

3. for $\mathrm{i}=1$ to $\mathrm{N}$ \{

$$
\begin{aligned}
& \text { if }((\mathrm{i} \Delta) \bmod \mathrm{N}=1) \mathrm{d}=\mathrm{i} \text {; } \\
& \quad / / \Delta \text { is relatively prime to } \mathrm{N} \text {. }
\end{aligned}
$$
break;

\section{\} \\ 4. $\mathrm{B}=\left(\mathrm{d}\left[\mathrm{A}_{\mathrm{ji}}\right]\right) \bmod \mathrm{N}$}

// $\mathrm{B}$ is the modular arithmetic inverse of $\mathrm{A}$

\}

\section{ILLUSTRATION OF THE CIPHER}

Let us consider the plaintext-Burn the forest as soon as the thieves enter into it. Let the key matrix $\mathrm{K}$ be taken in the form

$$
\mathrm{K}=\left(\begin{array}{rrrrrr}
99 & 4 & 12 & 9 & 5 & 12 \\
13 & 125 & 18 & 26 & 6 & 14 \\
30 & 10 & 124 & 24 & 26 & 87 \\
28 & 29 & 30 & 98 & 50 & 44 \\
63 & 45 & 78 & 89 & 120 & 56 \\
127 & 45 & 59 & 110 & 53 & 111
\end{array}\right)
$$

Here $n=6$.

Now let us focus our attention on the first six characters of the plaintext under consideration. The first six characters are Burn $-\mathrm{bt}$.

Considering the corresponding ASCII codes, the plaintext vector $\mathrm{p}$ can be obtained as

$$
\mathrm{p}=\left(\begin{array}{llllll}
66 & 117 & 114 & 110 & 32 & 116
\end{array}\right)^{\mathrm{T}} .
$$

Let us choose the inner key in the form

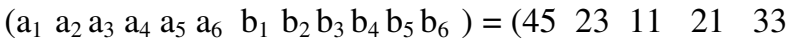

$\left.\begin{array}{lllllll}55 & 42 & 78 & 12 & 110 & 45 & 24\end{array}\right)$

On using (4.2) and (4.3), and the encryption algorithm 3.1 , we get

$$
\begin{aligned}
& \left(\begin{array}{lllllll}
\mathrm{P}_{1} \mathrm{P}_{2} \mathrm{P}_{3} \mathrm{P}_{4} \mathrm{P}_{5} \mathrm{P}_{6}
\end{array}\right)=\left(\begin{array}{llllll}
68 & 81 & 114 & 116 & 77 & 4
\end{array}\right) \\
& \text { and } \mathrm{C}=\left(\begin{array}{lllllll}
45 & 83 & 104 & 27 & 1 & 68
\end{array}\right)^{\mathrm{T}} .
\end{aligned}
$$

Then on using the algorithm 3.3, the modular arithmetic inverse of the matrix $\mathrm{K}$, denoted by $\mathrm{K}^{-1}$,is obtained as

$$
\mathrm{K}^{-1}=\left(\begin{array}{rrrrrr}
108 & 97 & 38 & 75 & 76 & 12 \\
88 & 68 & 98 & 57 & 114 & 66 \\
21 & 85 & 9 & 83 & 21 & 61 \\
6 & 95 & 54 & 68 & 13 & 28 \\
23 & 74 & 112 & 111 & 95 & 76 \\
26 & 110 & 91 & 46 & 118 & 96
\end{array}\right)
$$

It can be readily verified that $\mathrm{K}^{-1} \mathrm{~K} \bmod 128=\mathrm{K} \mathrm{K}^{-1}$ $\bmod 128=\mathrm{I}$.

Then on using the algorithm 3.2, we get

$\mathrm{P}=\left(\begin{array}{llllll}68 & 81 & 114 & 116 & 77 & 4\end{array}\right)^{\mathrm{T}}$

and $\left(\begin{array}{llllll}\mathrm{p}_{1} & \mathrm{p}_{2} & \mathrm{p}_{3} & \mathrm{p}_{4} & \mathrm{p}_{5} & \mathrm{p}_{6}\end{array}\right)=\left(\begin{array}{lllll}66 & 117 & 114 & 110 & 32\end{array}\right.$ $116)$.

The values of $\mathrm{p}_{1} \ldots \mathrm{p}_{6}$ given by (4.2) and (4.8) are the same. Thus we get back the plaintext.

\section{CRYPTANALYSIS}

In the process of encryption we have

$$
\mathrm{C}=\mathrm{KP} \bmod 128
$$

where $P_{i}=\left(a_{i} p_{i}+b_{i}\right) \bmod 128, i=1$ to $n$.

Here when the ciphertext $\mathrm{C}$ is known to us, the plaintext $p$ can be found if the matrix $K$, and the $a_{i}$ and 
the $b_{i}$ are known to us, i.e if the outer key and the inner key are known to us. The key matrix is of size $n \times n$. The $a_{i}$ 's are $n$ in number and the $b_{i}$ 's are also $n$ in number. The number of combinations of the secret key, including the outer key $\mathrm{K}$ and the inner key comprising $a_{i}$ and $b_{i}$, can be determined as follows. The outer key $K$ contains $\mathrm{n}^{2}$ numbers wherein each one can be represented in terms of 7 binary bits. Thus the key space corresponding to this key is given by

$2^{7 n^{2}}$. Now let us consider the key space of the inner key. As the $\mathrm{a}_{\mathrm{i}} \mathrm{s}$ are purely odd numbers, lying between 0 and 127 , they can be represented by 7 binary bits wherein the least significant bit all the while remain as 1. As the number of $a_{i} s$ is $n$, the possible number of combinations of the $a_{i} s$ is $2^{6 n}$. As $b_{i}$ is any number that lies between 0 and 127 , the possible number of combinations of $b_{i} s$ is $2^{7 n}$. Thus the total search space for the secret key including the outer key $\mathrm{K}$, and the inner key $a_{1}, a_{2} \ldots \ldots a_{n}, b_{1}, b_{2} \ldots \ldots b_{n}$ can be obtained as

$$
2^{7 n^{2}} \times 2^{6 n} \times 2^{7 n}=2^{7 n^{2}+13 n}
$$

For every possible key of the key space, the attacker has to find the plaintext till he gets a meaningful one. This process is to be carried out at least with half of the possible keys. If a plaintext corresponding to a secret key, can be obtained, by the decryption process in $10^{-7} \mathrm{sec}$, then the time $\mathrm{T}$ required for computing with half of the possible keys, is given by

$$
\mathrm{T}=\lambda \cdot 2^{7 \mathrm{n}^{2}+13 \mathrm{n}} \text { centuries }
$$

where $\lambda=10^{-7} / 60 \times 60 \times 24 \times 365_{\times} 100=1.585 \times 10^{-18}$

Let us now consider the known plaintext attack. In this case we know as many pairs of plaintext and ciphertext as we require. For each plaintext vector $\mathrm{p}$ we are to find $\mathrm{P} s$ for all possible combinations of the values of $a_{i}$ and $b_{i}$ i.e, for each possible inner key. Thus the number of $\mathrm{Ps}$ corresponding to each $\mathrm{p}$ is $\left(2^{6}\right)^{\mathrm{n}} \mathrm{x}$ $\left(2^{7}\right)^{\mathrm{n}}=2^{13 \mathrm{n}}$ as $\mathrm{a}_{\mathrm{i}}$ is an odd integer lying between 0 and 127 , and $b_{i}$ is any integer lying between 0 and 127 . If we take $n$ plaintext vectors and form a matrix with these vectors, then the number of matrices containing the corresponding Ps is $2^{13 n^{2}}$.

In view of the example given in section 4,we have $n=6$.In this case the number of matrices containing $\mathrm{P} s$ are $2^{13 \times 36}$.

Now, let us suppose that finding the modular arithmetic inverse of each matrix takes

$10^{-7}$ sec., then finding all possible modular arithmetic inverses will take

$$
2^{13 \times 36} \times 10^{-7} \approx 10^{133} \mathrm{sec} \approx 3.17 \times 10^{122} \text { centuries. }
$$

Thus we conclude that the strength of the cipher increases enormously, and it cannot be broken by the known plaintext attack.

\section{A LARGER BLOCK CIPHER USING INTERLACING AND ITERATION}

In section 2, we have developed the cipher for a block of $\mathrm{n}$ characters. Let us now extend the analysis for a block of $2 \mathrm{n}$ characters by introducing the concepts interlacing and iteration. In this section, the procedures used for encryption and decryption are presented in Figures 1 and 2.

In the procedure of encryption, the block consisting of $2 n$ characters is divided into two blocks left half and right half each containing $\mathrm{n}$ characters. On these two halves the same procedure, discussed in section 2 , is applied. Then we obtain $n$ characters as output on both the sides. The $\mathrm{n}$ characters on each side are converted into $7 \mathrm{n}$ binary bits. 


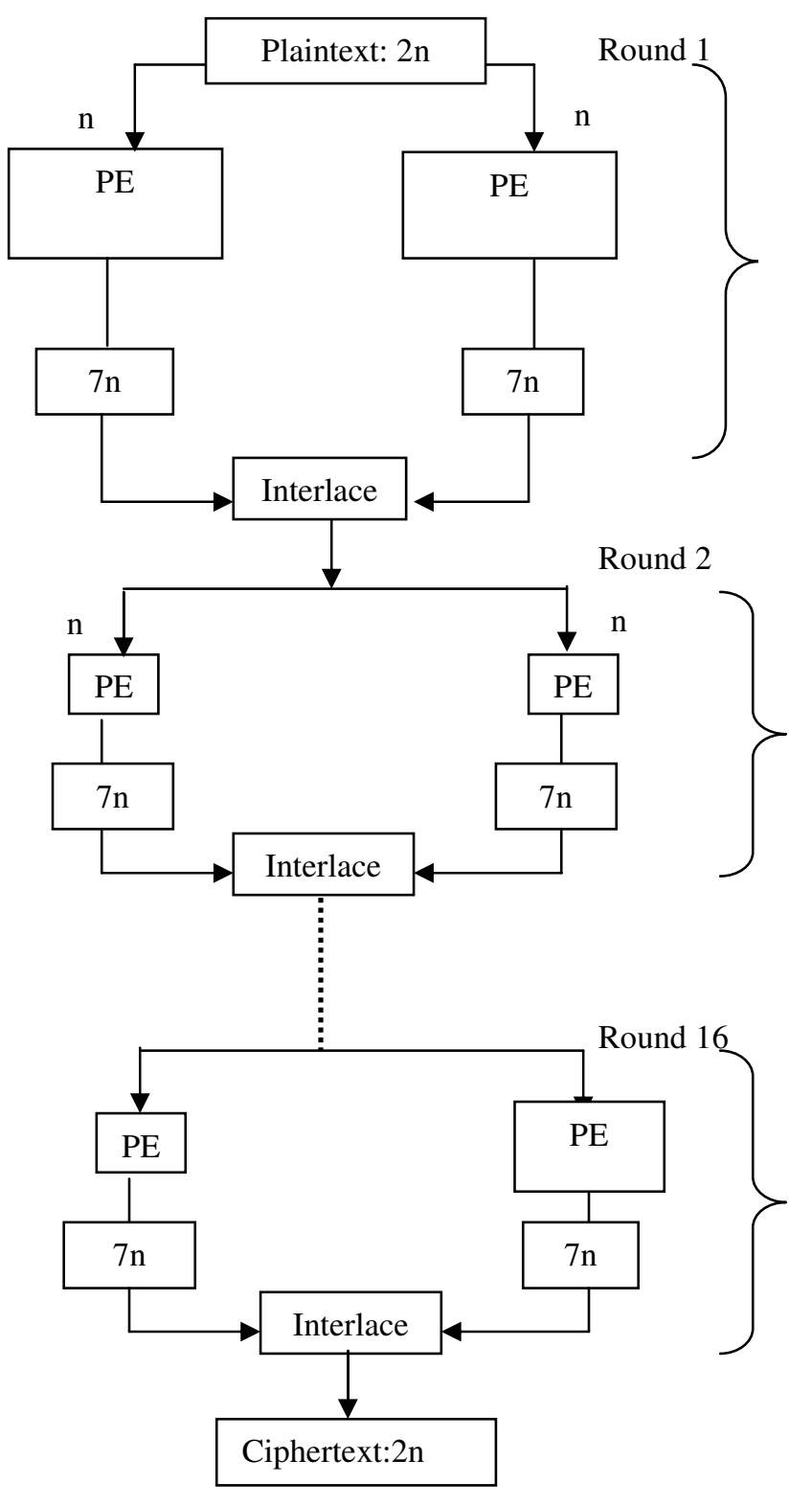

Fig.1: Procedure for Encryption in the case of larger block cipher containing $2 \mathrm{n}$
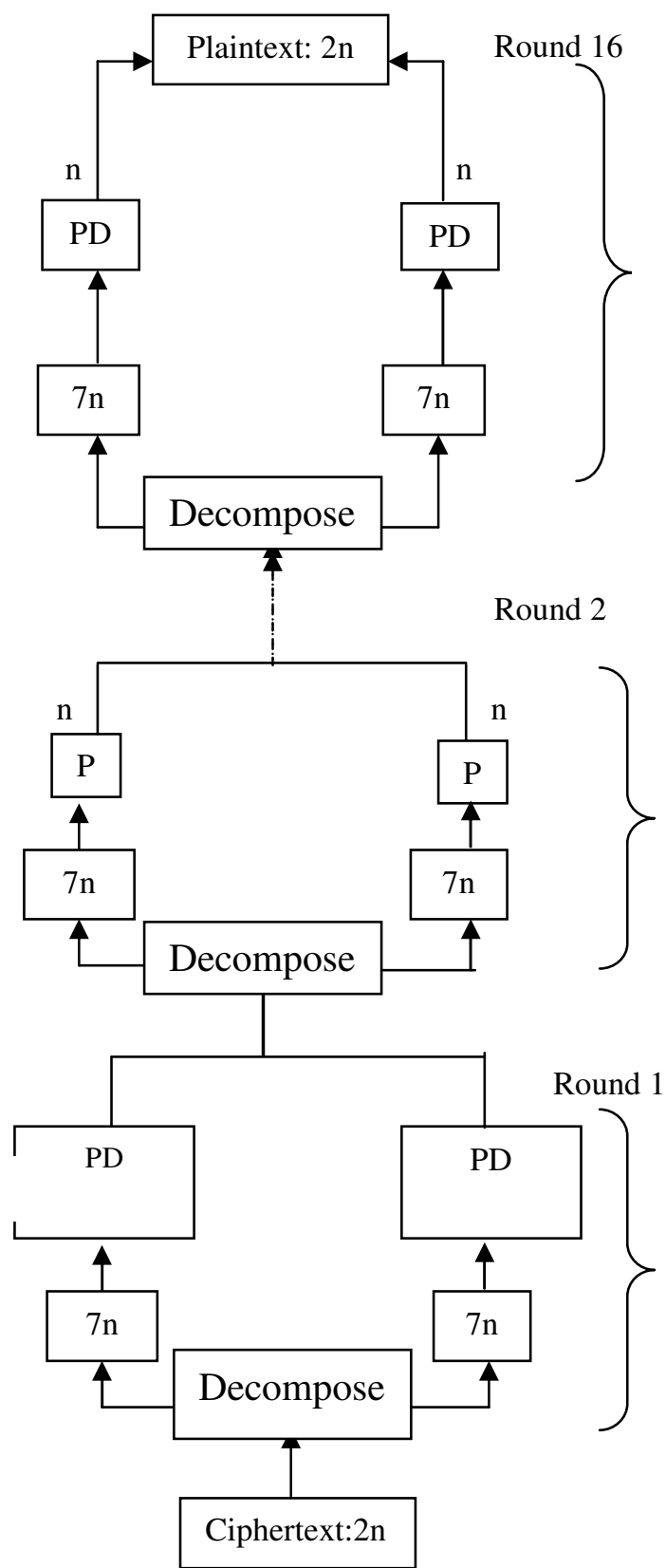

Fig.2: Procedure for Decryption in the case of larger block cipher containing $2 \mathrm{n}$ characters 
The process of interlacing can be described as follows. The first bit of left side $7 \mathrm{n}$ bits is placed as the first bit of an array. The first bit of the right side $7 \mathrm{n}$ bits is placed as a second bit in the array. Then the second bit of the left $7 \mathrm{n}$ bits is placed in the third place of the array. This process is continued till we exhaust all the $7 \mathrm{n}$ bits on both the sides.

The procedure described above constitutes one round. This process is repeated for 16 rounds and then ultimately we get the ciphertext.

The process of decryption is completely a reverse process of encryption. The decompose, used in decryption, is a function in which the $14 \mathrm{n}$ bits of the $2 \mathrm{n}$ characters are separated into $7 \mathrm{n}$ bits on the left side and $7 \mathrm{n}$ bits on the right side.

By using the process of encryption we are able to encrypt $2 \mathrm{n}$ characters and obtain the corresponding ciphertext. Further, by performing decryption we are able to obtain the plaintext of length $2 \mathrm{n}$ characters from the ciphertext.

In order to illustrate the above procedure, let us consider the plaintext

Burn to the for for

which is consisting of 12 characters. Following the encryption procedure given in Fig.1, the ciphertext corresponding to the plaintext (6.1), is obtained in terms of binary bits as follows

100011110010101000110111011100001001010111010 $100000011010001111101100101101000011101 \quad$ (6.2)

On applying the procedure given in Fig.2, the ciphertext (6.2) can be converted into the plaintext given by (6.1).

\section{AVALANCHE EFFECT}

Let us consider the plaintext given by (6.1). On changing the first character $\mathrm{B}$ in the plaintext to $\mathrm{C}$, the plaintext assumes the form

\section{Curn bithe bfor.}

In binary bit representation (6.1) and (6.3) differ only one in binary bit as B and C differ in one bit. Now on applying the encryption procedure given in Fig.1, on the modified plaintext (6.3), we get the ciphertext in the form

110100010011111111100100010001111001010101011 101111000110010111010011000101101100001

Here we notice that (6.2) and (6.4) differ in 30 bits. This indicates that the avalanche effect is not at all less significant.

Consider the effect on account of a change in the inner key, which plays a prominent role on the cipher. Let us now we take the inner key as $\begin{array}{llllllllllll}45 & 21 & 11 & 21 & 33 & 55 & 42 & 78 & 12 & 110 & 45 & 24\end{array}$ instead of (4.3). On using this key and the encryption procedure given in Fig.1, we get the ciphertext in the form 011010011101010101000111111000000101000000001 $001101110010010000001010011101011010000 \quad$ (6.6)

On comparing (6.2) and (6.6) we find that they differ in 31 bits. This again shows that the avalanche effect is significant.

\section{COMPUTATIONS AND CONCLUSIONS}

In this paper we have developed a block cipher by using linear congruences and modular arithmetic inverse. Firstly the plaintext vector is modified by using the linear congruences. These congruences contain a set of constants, which form a key called the inner key. The modified plaintext is operated by a key matrix called as outer key The inner and outer keys are used in the process of encryption and in the process of decryption, and they form the secret key. This cipher is extended to the case of larger block (block size is doubled) by introducing interlacing and iteration.

Here all the programs for encryption and decryption are written in $\mathrm{C}$ language. On performing computations we have obtained the ciphertext for a given plaintext and vice-versa. The ciphertext obtained in the case of the plaintext Burn the forest as soon as the thieves enter into it. is given by

100011110010101000110111011100001001010111010 100000011010001111101100101101000011101111101 010111101011000010110000000011010111010110001 001011101000110101001101100000100101110000001 001111011011111010011000010011101110000010001 001101001010000001100110101100000001100110110 000100100111011111100011001110110011001100111 000111111100001100100001101101100010110011110 000010100010000011110101111001101101100110101 010000111001010

From the cryptanalysis it is cleanly seen that the cipher cannot be broken by any cryptanalytic attack. The interlacing and the iteration strengthen the cipher remarkably.

From the analysis presented in this paper we find that the outer key and the inner key both play a vital role in strengthening the cipher. The strength is further enhanced in the case of the larger block.

\section{REFERENCES}

1. Cryptography and Network Security, William Stallings, $3^{\text {rd }}$ Edition, Pearson Education

2. On the Modular Arithmetic Inverse in the Cryptology of Hill cipher, 2005. V.U.K.Sastry, V.Janaki, Proceedings of North American Technology and Business Conference, Canada 